\title{
Extended Models for TSALBP with Ergonomic Risk Constraints *
}

\author{
J. Bautista * C. Batalla* R. Alfaro* A. Cano* \\ * Universitat Politècnica de Catalunya, Avda. Diagonal 647, 7th floor, \\ 08028 Barcelona, Spain (e-mail: \{joaquin.bautista; cristina.batalla; \\ rocio.alfaro; alberto.cano-perez\}@upc.edu).
}

\begin{abstract}
In this paper, we present an extension to the TSALB problem (Time and Space constrained Assembly Line Balancing) with ergonomic risk restrictions. This ergonomic risk is defined from the task durations and their categories regarding to the risk factor for the workers. A study is presented through a case linked to an engine assembly line in the Automobile industry, considering variable demand.
\end{abstract}

Keywords: Automobile industry, Ergonomics, Manufacturing systems, Mathematical models, Linear programming, Quality of work life.

\section{INTRODUCTION}

Circulating units are not identical in manufacturing systems with mixed-products assembly lines, like in the Automobile industry. This product variety means a variation in the use of resources (workers, tools, etc.) as well as in consumption of components. Therefore, the need for balancing an assembly line is present in its design. Obviously, in line balancing, if we talk about real situations, the technological and management constraints should be also taken into account.

The Assembly Line Balancing Problem $(A L B P)$ is a classic problem (Salveson, 1955) related to flow-oriented production systems. The problem deals to assign a set of elementary tasks (which may correspond to the assembly or disassembly of a product: motors, batteries, cars...) to a set of workstations or modules. The workstations are usually associated with teams of workers and/or robots, and they apply some of the work that will serve to complete the final product.

Typically, the workstations are arranged in a row, one behind another, and connected by a transport system, which allows movement of the work in progress at constant speed. Each workstation is given a constant time (cycle time, $c$ ) to complete the work that has been assigned.

Baybars (1986) divided the $A L B P$ into two classes: (1) Simple Assembly Line Balancing Problem $(S A L B P)$ and (2) General Assembly Line Balancing Problem (GALBP).

The $S A L B P$ class contains assembly problems that attempt to minimize the total idle time considering exclusively only two kinds of task assignment constraints:

- Cumulative constraints (associated with the available work time in the workstations).

* The authors greatly appreciate the collaboration of Nissan Spanish Industrial Operations (NSIO). This work was funded by project PROTHIUS-III, DPI2010-16759, including EDRF funding from the Spanish government.
- Precedence constraints (established by the order in which the tasks can be executed).

The GALBP class (Becker and Scholl, 2006) contains problems with additional considerations, as is the case in which the assignment of tasks is restricted (Scholl et al., 2010) or when certain tasks must be assigned in block (Battaïa and Dolgui, 2012). We find a new state of the art paper on Assembly Line Balancing Problems in (Battaïa and Dolgui, 2013).

By other hand, other problems from literature, with some limitations, are included in a family of problems under the name Time and Space constrained Assembly Line Balancing Problems (TSALBP) (Bautista and Pereira (2007); Chica et al. (2010); Chica et al. (2011)). These problems take into account elements, such as:

- the number of workstations $(m)$;

- the standard time assigned to each workstation $(c)$, which is calculated through an average of the processing times of all tasks according to the proportions, of each type of product, that are present in the demand plan, and;

- the available space or area $(A)$ to materials and tools at each workstation.

All types of problems discussed above, consist on assigning a set of tasks to a set of workstations. In many cases, these workstations are associated with workers, so in addition to the technological and management constraints, ergonomics must be contemplated.

One of the main objectives of the ergonomics is to adapt the operations that the workers must perform to guarantee their safety, welfare and to improve their efficiency.

Although the problems of a poor ergonomic design of a workplace affect all areas of employment, manufacturing is one of the most affected. Specifically, in the case of manufacturing assembly lines with mixed products, the ergonomic risk is present at workstations and may affect the performance of workers and the line. 
In such environments, ergonomic risk is given basically by the components related to somatic comfort and psychological comfort.

The somatic comfort determinates the set of physical demands to which a worker is exposed throughout the working day. To analyze this type of ergonomic risk, three factors, among others, can be analyzed. These are:

- Postural load: Throughout the workday the workers may adopt, repeatedly, inappropriate or awkward postures that can result long term in fatigue and musculoskeletal disorders (McAtamney and Corlett, 1993).

- Repetitive movements: A workstation may involve a set of repeated upper-limb movements by the worker. This may cause long term musculoskeletal injuries (Bao et al., 2009).

- Manual handling: Some tasks involve the lifting, moving, pushing, grasping and transporting objects (Waters et al., 1997).

By the other hand, the psychological comfort refers to the set of necessary mental conditions that the workers must have to develop their tasks. These conditions are: autonomy, social support, acceptable workloads and a favorable work environment. To evaluate this component of ergonomic risk we have several methods, as the COPSOQ (Copenhagen Psychosocial Questionnaire) that was adapted and validated in Spain with the name $I S$ TAS21, the LEST method developed by the "Laboratoire d'Economie et Sociologie du Travail" and other methods with less reliability.

Our proposal is to incorporate into the TSALBP or in other assembly line problems the factors that imply these ergonomic problems.

This paper is organized as follows: In Section 2, using as reference the $T S A L B P$ models present in the literature, we incorporate ergonomics, obtaining new models that consider technological, managerial and ergonomic factors. In Section 3 we compare, through an example, the offered results by the proposed models with those provided by the models to $S A L B P$ and TSALBP. In Section 4 we describe the computational experience carried out and the results obtained though a case study linked to the Nissan powertrain plant in Barcelona. Finally, in Section 5 we show the conclusions of the study.

\section{INCORPORATING ERGONOMICS INTO THE TSALBP}

\subsection{The TSALBP}

Given a set $J$ of $|J|$ tasks with their temporal, $t_{j}$, and spatial, $a_{j}$, attributes $(\forall j=1, \ldots,|J|)$ and a precedence graph, each task must be assigned to a single station $k$ $(k=1, \ldots, m)$, such that: (1) all the precedence constraints are satisfied, (2) no workstation workload time is greater than the cycle time $(c)$ and (3) no area required by the station is greater than the available area per station $(A)$.

Then, depending on the elements $m, c$ and $A$, we have eight types of problems, according to the objective of each one of them. First, we have a feasibility problem $(T S A L B P-F)$, which objective is to satisfy the constraints of the number of workstations, the standard time assigned to workstations to process any product and the available space. Second, we have three mono-objective problems focused on minimizing the number of workstations, the cycle time or the required area by the workstations $(T S A L B P-1$, $T S A L B P-2$ and $T S A L B P-3$, respectively). Finally, we have four multi-objective problems focused on minimizing two of the three elements, the number of workstations and the cycle time $(T S A L B P-1 / 2)$, the number of workstations and the required space $(T S A L B P-1 / 3)$, the cycle time and the required space $(T S A L B P-2 / 3)$ or the three elements at once $(T S A L B P-1 / 2 / 3)$.

As an example, bellow we can see the mathematical model for the $T S A L B P-1$ :

$$
\text { Min } z_{1}=m
$$

Subject to:

$$
\begin{array}{ll}
m-\sum_{k=1}^{m_{\max }} k x_{j, k} \geq 0 & (j=1, \ldots,|J|) \\
\sum_{j=1}^{|J|} t_{j} x_{j, k} \leq c & \left(k=1, \ldots, m_{\max }\right) \\
\sum_{j=1}^{|J|} a_{j} x_{j, k} \leq A & \left(k=1, \ldots, m_{\max }\right) \\
\sum_{k=1}^{m_{\max }} x_{j, k}=1 & (j=1, \ldots,|J|) \\
\sum_{k=1}^{m_{\max }} k\left(x_{j, k}-x_{i, k}\right) \geq 0 & \left(j \leq i, j \leq|J|: i \in P_{j}\right) \\
x_{j, k} \in\{0,1\} \quad(j=1, \ldots,|J|) \wedge\left(k=1, \ldots, m_{\max }\right)
\end{array}
$$

Where, $x_{j, k}$ is a binary variable that is equal to 1 if a task $j(j=1, \ldots,|J|)$ is assigned to the workstation $k$ $\left(k=1, \ldots, m_{\max }\right)$, and to 0 otherwise; $P_{j}$ is a parameter that indicates the set of direct precedent tasks of the task $j$ $(j=1, \ldots,|J|)$ and the objective is minimizing the number of workstations $\left(m \leq m_{\max }=|K|\right)$.

\subsection{The TSALBP with ergonomics}

Otto and Scholl, (2011) employ several techniques to incorporate the ergonomic risks in the $S A L B P-1$.

In a first approximation, given the set $K$ of stations, to each workload, $S_{k}$, assigned at workstation $k(k=$ $1, \ldots,|K|)$, is determined an ergonomic risk, $F\left(S_{k}\right)$. Moreover, a maximum value, called $E r g$, is established for this ergonomic risk. Consequently, they can add to the original models the following constraints (8), satisfying: $F\left(S_{k}\right) \leq F\left(S_{k} \cup\{j\}\right) \forall S_{k} \forall j \in J$.

$$
F\left(S_{k}\right) \leq \operatorname{Erg} \quad \forall k=1, \ldots,|K|
$$

Alternatively to the conditions (8), they propose the ErgoSALBP-1, adding to the $S A L B P-1$ a new objective function composed by two terms:

$$
\left.\operatorname{Min} \quad K^{\prime}(x)=K(x)\right)+\omega \cdot \xi\left(F\left(S_{k}\right)\right)
$$


Where $K(x)$ is the number of workstations; $\omega$ is a nonnegative weight and $\xi\left(F\left(S_{k}\right)\right)$ is a function that includes the ergonomic risk factors, $F\left(S_{k}\right), \forall k$.

Logically, the constraints (8), presented by Otto and Scholl, (2011), can be extended if we take into account, in the line design, a minimum and maximum value to the ergonomic risk and the different factors that can mean ergonomic risks.

Indeed, if we also consider that the methods of risk assessment (OCRA Check-List, NIOSH, RULA) classify the activities or tasks in different categories (e.g. from 1 to 4) depending on the level of risk involved, we have:

$\Phi$ Set of ergonomic risk factors (physical, psychics, physical-psychic).

$X_{\phi}$ Set of categories for the ergonomic risk factor $\phi \in$ $\Phi$. When only one risk factor is analyzed, we will call $X$ to the set of categories. The set of categories for one ergonomic risk indicates the intensity, effort or difficulty that this factor can present.

$J \quad$ Set of elemental tasks.

$j \quad$ Index of tasks $j \in J(j=1, \ldots,|J|)$.

$\chi_{\phi, j}$ Category of the task $j \in J\left(\chi_{\phi, j} \in X_{\phi}\right)$ associated to the risk factor $\phi \in \Phi$, that is a non-negative integer value. To one risk factor we will have $\chi_{j}$.

Thus, we can define:

- The ergonomic risk of the task $j \in J$ regarding the ergonomic risk factor $\phi \in \Phi$ :

$$
F_{\phi, j}=t_{j} \chi_{\phi, j} \quad(\forall \phi \in \Phi ; \forall j \in J)
$$

- The ergonomic risk of the subset of task $J_{0} \subseteq J$ regarding the ergonomic risk factor $\phi \in \Phi$ :

$$
F_{\phi}\left(J_{0}\right)=\sum_{j \in J_{0}} t_{j} \chi_{\phi, j} \quad\left(\forall \phi \in \Phi ; \forall J_{0} \subseteq J\right)
$$

where, $J_{0}$ can be a task, an operation that adds a set of elementary task, a meta-operation that adds a set of operations or the workstation workload.

- The processing time of the subset of task $J_{0} \subseteq J$ :

$$
t\left(J_{0}\right)=\sum_{j \in J_{0}} t_{j} \quad\left(\forall J_{0} \subseteq J\right)
$$

- The category of the subset of task $J_{0} \subseteq J$ regarding the ergonomic risk factor $\phi \in \Phi$ :

$$
\chi_{\phi}\left(J_{0}\right)=\frac{F_{\phi}\left(J_{0}\right)}{t\left(J_{0}\right)} \quad\left(\forall \phi \in \Phi ; \forall J_{0} \subseteq J\right)
$$

Where $\chi_{\phi}\left(J_{0}\right)$ is a real value.

- The corrected category of the subset of task $J_{0} \subseteq J$ for the ergonomic risk factor $\phi \in \Phi$ :

$$
\hat{\chi}_{\phi}\left(J_{0}\right)=\left\lceil\chi_{\phi}\left(J_{0}\right)\right\rceil \quad\left(\forall \phi \in \Phi ; \forall J_{0} \subseteq J\right)
$$

Therefore, our proposal is to incorporate into the TSALBP1 models the restrictions associated to ergonomic risk. Particularly, if we consider $J_{0}$ as a workstation from the set of workstations, $K$, therefore we have $F_{\phi}\left(S_{k}\right), t\left(S_{k}\right)$, $\chi_{\phi}\left(S_{k}\right)$ and $\hat{\chi}_{\phi}\left(S_{k}\right) \forall k \in K$, we will impose the following limitations to to each station and risk factor:

(1) Upper and lower limitation of actual ergonomic risk:

$$
F_{\phi}^{\min } \leq t\left(S_{k}\right) \chi_{\phi}\left(S_{k}\right) \leq F_{\phi}^{\max } \quad(\forall k \in K ; \forall \phi \in \Phi)
$$

(2) Upper and lower limitation of corrected ergonomic risk:

$$
F_{\phi}^{\text {min }} \leq t\left(S_{k}\right) \hat{\chi}_{\phi}\left(S_{k}\right) \leq F_{\phi}^{\max } \quad(\forall k \in K ; \forall \phi \in \Phi)
$$

Consequently, considering the limitations (15) or (16) we have new models to the TSALBP:

- $T S A L B P-1$ with upper and lower limitation of actual ergonomics risk:

$$
\text { Min } z_{1}=m
$$

Subject to: $(2)-(7)$ from $T S A L B P-1$

$$
F_{\phi}^{\text {min }} \leq \sum_{j=1}^{|J|} t_{j} \chi_{\phi, j} x_{j, k} \leq F_{\phi}^{\max }(\forall k \in K ; \forall \phi \in \Phi)
$$

- $T S A L B P-1$ with upper and lower limitation of corrected ergonomics risk:

$$
\text { Min } z_{1}=m
$$

Subject to: $(2)-(7)$ from $T S A L B P-1$

$$
F_{\phi}^{\text {min }} \leq \sum_{j=1}^{|J|} t_{j} \hat{\chi}_{\phi, j} x_{j, k} \leq F_{\phi}^{\max }(\forall k \in K ; \forall \phi \in \Phi)
$$

where $F_{\phi}^{\text {min }}$ and $F_{\phi}^{\max }$ are the limits of the ergonomic risk to the risk factor $\phi \in \Phi$. Obviously, the above models, proposed for elemental tasks $(j \in J)$, may arise for subsets of elemental tasks $\left(J_{0} \subseteq J\right)$.

If we consider the new constraints (18) or (20) and the set of human aspects that we can observe $H$, in our case: the soma aspect $(\sigma)$, the psique aspect $(\varphi)$ or both $(\sigma \cup \varphi)$; we can find a new classification for the TSALBP, that is the TSALBP_erg (see table 1):

Table 1. TSALBP_erg typology.

\begin{tabular}{lcccc} 
Name & $\mathrm{m}$ & $\mathrm{c}$ & $\mathrm{A}$ & Type \\
\hline TSALBP-F- $\eta$ & Given & Given & Given & $\mathrm{F}$ \\
TSALBP-1- $\eta$ & Minimize & Given & Given & OP \\
TSALBP-2- $\eta$ & Given & Minimize & Given & OP \\
TSALBP-3- $\eta$ & Given & Given & Minimize & OP \\
TSALBP-1/2- $\eta$ & Minimize & Minimize & Given & MOP \\
TSALBP-1/3- $\eta$ & Minimize & Given & Minimize & MOP \\
TSALBP-2/3- $\eta$ & Given & Minimize & Minimize & MOP \\
TSALBP-1/2/3- $\eta$ & Minimize & Minimize & Minimize & MOP \\
\hline
\end{tabular}

In table 1 the suffixes 1,2 , and 3 refer to the minimization of $m, c$ and $A$, respectively. The suffix $F$ refers to a feasibility problem. The post-suffix $\eta$ refers to the type of the restriction linked to the human aspects, psychic and somatic, being the element $\eta \in H$ where $H=\{\emptyset, \sigma, \varphi, \sigma \cup$ $\varphi\}$. The column "Type" indicates if the problem is one of feasibility (F), mono-objective (OP) or multi-objective (MOP).

\section{AN EXAMPLE}

To illustrate the effect of incorporating additional constraints into the assembly line balancing problems, below we present an example for three types of these problems: the $S A L B P-1$, the TSALBP-1 and the TSALBP-1- $\sigma$. 
Given a set of eight tasks $(|J|=8)$, whose operation times, $t_{j}(j=1, \ldots,|J|)$, required space, $a_{j}(j=1, \ldots,|J|)$, category for the physical factor, $\chi_{j}(j=1, \ldots,|J|)$, and precedence graph (see Fig. 1), each task must be assigned to a single stations such that: (1) no station workload time is greater than the cycle time, $c=20 s$; (2) no area required by a station is greater than the available lineal area per station, $A=20 \mathrm{dm}$; and (3) no station with ergonomic risk for physical factor greater than the maximum allowed, $F^{\max }=60 e-s$ (ergo-seconds).

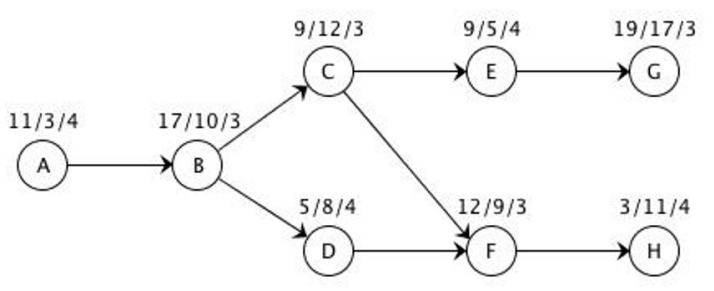

Fig. 1. Precedence graph of tasks. At each vertex we can see the tuple $t_{j} / a_{j} / \chi_{j}$ corresponding to the task.

If we considerer the $S A L B P-1$, that minimizes the number of workstations, $m$, given a fixed value of the cycle time $(c=20 s)$, we obtain 5 stations as solution (see Fig. 2).

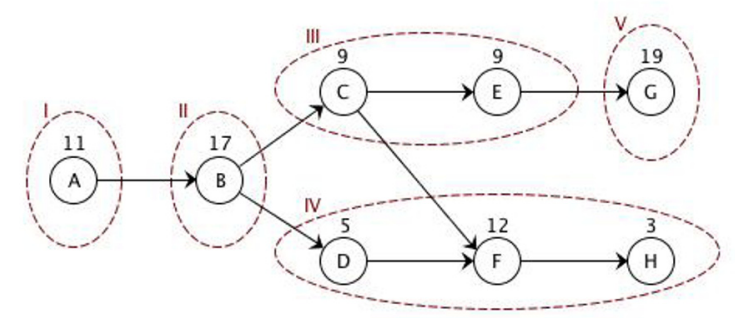

Fig. 2. Solution obtained by $S A L B P-1(m=5)$.

On the other hand, if we take into account the TSALBP1 , that minimizes the number of workstations, $m$, given a fixed value of the cycle time $(c=20 s)$ and the available space for each workstation $(A=20 \mathrm{dm})$, we can see that the obtained number of workstations is greater than the obtained by the $S A L B P-1$, in one unit (see Fig. 3 ).

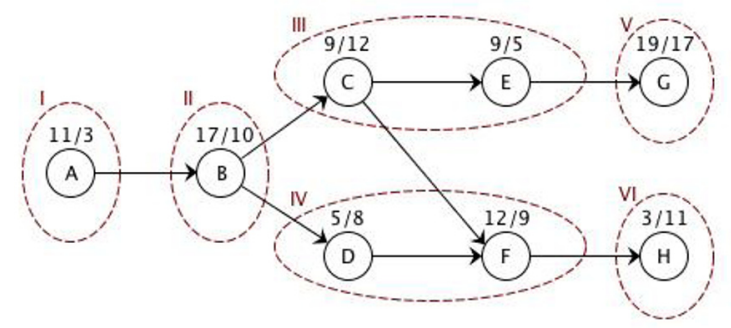

Fig. 3. Solution obtained by TSALBP-1 $(m=6)$.

Finally, if we consider the risk factor category, $\chi_{j}$, and the operation time, $t_{j}$, as shown in equation (10), we can obtain the ergonomic risk associated to each task, $F_{j}$ $(j=1, \ldots,|J|)$ (see table 2$)$.
Table 2. Ergonomic risk.

\begin{tabular}{ccccccccc} 
Tasks & $\mathrm{A}$ & $\mathrm{B}$ & $\mathrm{C}$ & $\mathrm{D}$ & $\mathrm{E}$ & $\mathrm{F}$ & $\mathrm{G}$ & $\mathrm{H}$ \\
\hline$t_{j}$ & 11 & 17 & 9 & 5 & 9 & 12 & 19 & 3 \\
$\chi_{j}$ & 4 & 3 & 3 & 4 & 4 & 3 & 3 & 4 \\
\hline$F_{j}$ & 44 & 51 & 27 & 20 & 36 & 36 & 57 & 12 \\
\hline
\end{tabular}

Then, considering the equation (18) we can solve the $T S A L B P-1-\sigma$, that minimizes the number of workstations, $m$, given a fixed value of the cycle time $(c=20 s)$, the available space for each workstation $(A=20 \mathrm{dm})$ and the maximum ergonomic risk for each workstation $\left(F^{\max }=60 e-s\right)($ see Fig. 4$)$.

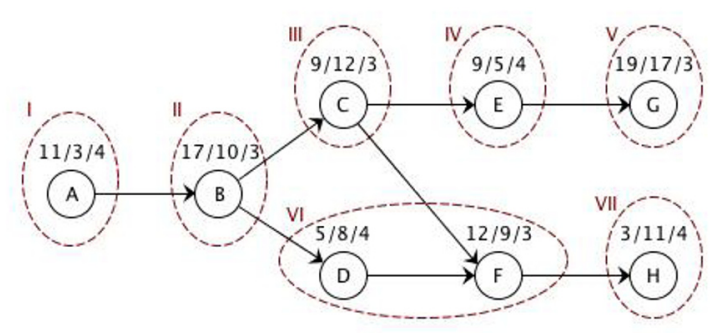

Fig. 4. Solution obtained by TSALBP-1- $\sigma(m=7)$.

As we can see from the example, depending on the limiting factors that we consider, the resulting number of stations will be one or other. Obviously, a greater number of conditional factors means a greater number of workstations. In the solution offered by the TSALBP-1- $\sigma$, we note that only two tasks can be grouped in a single station $\left(F\left(S_{V I}\right)=F_{D}+F_{F}=20+36=56 \leq 60 e-s\right)$, obtaining a solution of 7 workstation.

\section{COMPUTATIONAL EXPERIENCE}

To evaluate the influence of ergonomic risk constraints on the number of workstations of the line and their configuration, we solve the models $S A L B P-1$, TSALBP1 and the $T S A L B P-1-\sigma$ with upper limitation of actual ergonomics risk through a case study that corresponds to an assembly line from Nissan's plant in Barcelona.

The assembly line on which we perform the study is an assembly line of mixed products. Specifically, in this line are assembled nine types of engines $\left(p_{1}, \ldots, p_{9}\right)$ with different destinations and assembly features. These types of engines are grouped into three classes: $4 \mathrm{x} 4\left(p_{1}, \ldots, p_{3}\right)$; vans $\left(p_{4}, p_{5}\right)$; trucks $\left(p_{6}, \ldots, p_{9}\right)$. Logically, given a global demand, the demand for each one of the nine types of engines is not homogeneous among time and is not equal for each one. Therefore, although the daily production capacity is kept as constant (270 engines), the line must be able to adapt to different demand plans based on partial demands of each engine type. For this reason, several instances corresponding to different production mixes, have been used to solve the 3 problems studied in this document, $S A L B P-1$, TSALBP-1 and TSALBP- $\sigma$. Each one of these production programs corresponds to a set of average times of operation (Chica et al., 2012), weighted by the demand of the nine types of engines. In short, the change in production mix affects the weighted duration of each operation involved in the process and therefore may require a rebalancing of the line. 
In this study we select nine production mixes (see table 3 ) from the 23 available instances (see table 7 on Bautista et al. (2012)). The 23 instances for the block I are equivalent to the other 23 of the block II, because the proportions of engine types are maintained on both blocks respectively, changing only the total demand. Selected the nine production mixes, we will determine the processing times of the 140 operations as a function of each mix.

Table 3. Production units of the engine types for each production plan.

\begin{tabular}{c|c|ccccccccc} 
& & \multicolumn{10}{|c}{ Production plans } \\
Family & & 1 & 2 & 3 & 6 & 9 & 10 & 11 & 12 & 18 \\
\hline $4 \times 4$ & $p_{1}$ & 30 & 30 & 10 & 50 & 70 & 10 & 10 & 24 & 60 \\
& $p_{2}$ & 30 & 30 & 10 & 50 & 70 & 10 & 10 & 23 & 60 \\
& $p_{3}$ & 30 & 30 & 10 & 50 & 70 & 10 & 10 & 23 & 60 \\
\hline VAN & $p_{4}$ & 30 & 45 & 60 & 30 & 15 & 105 & 15 & 45 & 30 \\
& $p_{5}$ & 30 & 45 & 60 & 30 & 15 & 105 & 15 & 45 & 30 \\
\hline trucks & $p_{6}$ & 30 & 23 & 30 & 15 & 8 & 8 & 53 & 28 & 8 \\
& $p_{7}$ & 30 & 23 & 30 & 15 & 8 & 8 & 53 & 28 & 8 \\
& $p_{8}$ & 30 & 22 & 30 & 15 & 7 & 7 & 52 & 27 & 7 \\
& $p_{9}$ & 30 & 22 & 30 & 15 & 7 & 7 & 52 & 27 & 7 \\
\hline
\end{tabular}

There will be a single grouping of operations to metaoperations. That is, the 140 operations (related to the 378 elementary tasks) (Chica et al. (2010)) will become 36 meta-operations. For us, a meta-operation is a set of aggregate operations taking into account that there is a cycle time of $180 \mathrm{~s}$, an available longitudinal area of $400 \mathrm{~cm}$ and considering the links of precedence and/or succession.

Each one of the nine resulting instances will be solved, using the CPLEX solver v11.0 (single-processor license) running on a MacPro computer with an Intel Xeon 3.0 $\mathrm{GHz} \mathrm{CPU}$ and $2 \mathrm{~GB}$ RAM using Windows XP without any limit on the CPU time.

In table 4 , we can see the number of workstations obtained for each problem. The results show how adding constraints on models influences the obtained number of stations. Thus, we see that if we consider only the cycle time constraint, $c=180 \mathrm{~s}$, we get a number of workstations from 19 to 20, depending on the production plan used. Moreover, if we also consider, when the meta-operations are assigned to a station, the maximum available area of $400 \mathrm{~cm}$, the number of stations is renumbered 21, increasing by 1 or 2 stations depending on the mix. Finally, if, in addition to the cycle time and the maximum available area, we consider that the set of meta-operations assigned to a station can not exceed a maximum ergonomic risk of $465 e-s$ we have as result 22 and 23 workstations.

Table 4. Number of workstations obtained by $S A L B P-1, T S A L B P-1$ and TSALBP-1- $\sigma$ with a fixed values of cycle time $(c=180 \mathrm{~s})$, available space $(A=400 \mathrm{~cm})$ and ergonomic risk $\left(F^{\max }=465 e-s\right)$ to each station.

Production plans

\begin{tabular}{lccccccccc} 
& 1 & 2 & 3 & 6 & 9 & 10 & 11 & 12 & 18 \\
\hline SALBP-1 & 19 & 20 & 20 & 19 & 19 & 20 & 20 & 20 & 19 \\
TSALBP-1 & 21 & 21 & 21 & 21 & 21 & 21 & 21 & 21 & 21 \\
TSALBP $-1-\sigma$ & 22 & 23 & 23 & 23 & 23 & 23 & 22 & 23 & 23 \\
\hline
\end{tabular}

Table 5. Workstations Configuration $\left(k_{M i x_{\#}}=\right.$ $1, \ldots, m)$ resulting from $S A L B P-1, T S A L B P-1$ and TSALBP-1- $\sigma$ with $c=180 \mathrm{~s}, A=400 \mathrm{~cm}$ and $F^{\max }=360 e-s$ to mixes 1 and 6 .

\begin{tabular}{c|ll|ll|ll} 
Meta & \multicolumn{2}{|c|}{$S A L B P-1$} & \multicolumn{2}{|c|}{$T S A L B P-1$} & \multicolumn{2}{c}{$T S A L B P-1-\sigma$} \\
operation & $k_{\text {Mix } 1}$ & $k_{\text {Mix } 6}$ & $k_{\text {Mix } 1}$ & $k_{\text {Mix } 6}$ & $k_{M i x 1}$ & $k_{M i x 6}$ \\
\hline 1 & 1 & 1 & 1 & 1 & 1 & 1 \\
2 & 2 & 2 & 2 & 2 & 2 & 2 \\
3 & 3 & 3 & 3 & 3 & $\mathbf{6}$ & $\mathbf{3}$ \\
4 & 3 & 3 & 3 & 3 & 3 & 3 \\
5 & 4 & 4 & 4 & 4 & 4 & 4 \\
6 & 5 & 5 & 5 & 5 & 5 & 5 \\
7 & 5 & 5 & 5 & 5 & 5 & 5 \\
8 & 5 & 5 & 6 & 6 & 6 & 6 \\
9 & 19 & 19 & 6 & 6 & $\mathbf{1 3}$ & $\mathbf{6}$ \\
10 & 6 & 6 & 7 & 7 & $\mathbf{6}$ & $\mathbf{7}$ \\
11 & 6 & 6 & 7 & 7 & 7 & 7 \\
12 & 6 & 6 & 8 & 8 & 8 & 8 \\
13 & 7 & 7 & 8 & 8 & 8 & 8 \\
14 & 7 & 7 & 9 & 9 & 9 & 9 \\
15 & 8 & 8 & 9 & 9 & 10 & 10 \\
16 & 8 & 8 & 10 & 10 & 11 & 11 \\
17 & 9 & 9 & 10 & 10 & 12 & 12 \\
18 & 9 & 9 & 11 & 11 & 12 & 13 \\
19 & 10 & 10 & 11 & 11 & 13 & 13 \\
20 & 10 & 10 & 12 & 12 & 14 & 14 \\
21 & 11 & 11 & 13 & 13 & 15 & 15 \\
22 & 11 & 11 & 14 & 14 & 16 & 16 \\
23 & 12 & 12 & 14 & 14 & 16 & 16 \\
24 & 12 & 12 & 15 & 15 & 17 & 17 \\
25 & $\mathbf{1 7}$ & $\mathbf{1 6}$ & 15 & 15 & $\mathbf{1 7}$ & $\mathbf{2 4}$ \\
26 & 19 & 19 & 15 & 15 & $\mathbf{2 1}$ & $\mathbf{2 4}$ \\
27 & 13 & 13 & 16 & 16 & 18 & 18 \\
28 & 14 & 14 & $\mathbf{1 6}$ & $\mathbf{1 7}$ & 19 & 19 \\
29 & 14 & 14 & 17 & 17 & 19 & 19 \\
30 & 14 & 14 & 17 & 17 & $\mathbf{1 9}$ & $\mathbf{2 0}$ \\
31 & 15 & 15 & 18 & 18 & 20 & 20 \\
32 & 15 & 15 & 18 & 18 & $\mathbf{2 1}$ & $\mathbf{2 0}$ \\
33 & 16 & 16 & 19 & 19 & $\mathbf{2 2}$ & $\mathbf{2 1}$ \\
34 & 16 & 16 & 19 & 19 & $\mathbf{2 2}$ & $\mathbf{2 1}$ \\
35 & 17 & 17 & 20 & 20 & $\mathbf{2 3}$ & $\mathbf{2 2}$ \\
36 & 18 & 18 & 21 & 21 & $\mathbf{2 4}$ & $\mathbf{2 3}$ \\
\hline$m$ & 19 & 19 & 21 & 21 & 24 & 24 \\
\hline & & & & & &
\end{tabular}

We have seen in table 4 that the number of stations depends on the composition of the production mix (e.g. for Mix 1 a result of 19 workstations is obtained for the $S A L B P-1$ and 22 for the TSALBP-1- $\sigma$, while the Mix 2, gives a solution of 20 and 23 workstations, respectively). Similarly, although we can see the same result regarding the number of workstations in different production plans, the workstation configuration is not necessarily the same. Table 5 shows that the station assignment of each metaoperation, obtained by the three problems respectively, is different for two different production programs, the Mix 1 and Mix6, given a maximum ergonomic risk of $2 c=360 e-s$. For example, in the case of $S A L B P-1$, we can see that for Mix 1, the meta-operation 25 is assigned to station $k=17$, while this meta-operation is assigned to workstation $k=16$ for the Mix6. In TSALBP-1, occurs the same for the meta-operation 28, being assigned to station $k=16$ for $M i x 1$ and in the workstation $k=17$ for the Mix6. Finally, for the TSALBP-1- $\sigma$, the line configuration presents more differences depending on the mix, varying the workload assigned to the stations $3,6,7,13,17,19$, 20, 21, 22, 23 and 24 . 
Finally, if we look only at the $T S A L B P-1-\sigma$, we conclude that the configuration of the line depends on the maximum ergonomic risk that is allowed to each workstation. In table 6 , we can see that the number of stations is different for several intervals of maximum ergonomic risk. In particular, we observe that for a maximum allowable ergonomic risk of $360 e-s$ (which is equivalent to a maximum level of intensity or category for the risk factor of 2 for all the workstations) the number of workstations is 24 for all studied demand plans. However, when the maximum ergonomic risk is greater the number of stations decreases (e.g. for an $F^{\max }=480 e-s$ is obtained 22 workstations for each mix). Moreover, we note that the variation in the number of stations is not homogeneous for all production programs, obtaining a different number of stations for certain maximum risk levels (e.g. with $F^{\max }=465 e-s$ we obtain 22 stations for the production plans 1 and 11 , and 23 stations for the rest, with $F^{\max }=525 e-s$ are required 21 stations for the mixes 1 and 11 , and 22 for the rest, and $F^{\max }=530 e-s$ requires 21 workstations for all production plans, except for the mix 10 that requires 22 stations).

Table 6. Number of workstations obtained by $T S A L B P-1-\sigma$ with a cycle time of $180 \mathrm{~s}$, an available space of $400 \mathrm{~cm}$ and different maximum ergonomic risks.

\begin{tabular}{c|ccccccccc}
$F^{\max }$ & \multicolumn{10}{|c}{ Production plans } \\
& 1 & 2 & 3 & 6 & 9 & 10 & 11 & 12 & 18 \\
\hline 360 & 24 & 24 & 24 & 24 & 24 & 24 & 24 & 24 & 24 \\
450 & 24 & 24 & 24 & 24 & 24 & 24 & 24 & 24 & 24 \\
460 & 24 & 24 & 24 & 24 & 24 & 23 & 24 & 24 & 24 \\
465 & 22 & 23 & 23 & 23 & 23 & 23 & 22 & 23 & 23 \\
480 & 22 & 22 & 22 & 22 & 22 & 22 & 22 & 22 & 22 \\
510 & 22 & 22 & 22 & 22 & 22 & 22 & 22 & 22 & 22 \\
525 & 21 & 22 & 22 & 22 & 22 & 22 & 21 & 22 & 22 \\
530 & 21 & 21 & 21 & 21 & 21 & 22 & 21 & 21 & 21 \\
540 & 21 & 21 & 21 & 21 & 21 & 21 & 21 & 21 & 21 \\
\hline
\end{tabular}

\section{CONCLUSION}

In this paper we proposed several variants to the $T S A L B$ problem, incorporating ergonomic risk constraints.

The case study considered corresponds to an engine assembly line of Nissan plant in Barcelona and focuses on the analysis of the variation of the required number of workstations for balancing the line and the variation of the assigned tasks to each workstation, regarding two aspects: the engine demand variation and the variation of the allowed maximum ergonomic risk. The obtained result allowed us to observe that the variation of engine mix (with a constant total demand equal to 270 engines) influences the human resource and space requirements, increasing both requirements by $5 \%$ respect the initial situation, where the demand of engine types was balanced. On the other hand, the ergonomic risk reduction supposes a human and space resource increase around 15\%. Both the mix variation and the risk reduction require that the workstations are rearranged with the consequent operation costs and worker training.

\section{ACKNOWLEDGEMENTS}

The authors greatly appreciate the collaboration of Nissan Spanish Industrial Operations (NSIO). This work was funded by project PROTHIUS-III, DPI2010-16759, including EDRF funding from the Spanish government.

\section{REFERENCES}

Bao, S., Howard, N., Silverstein, B. (2009). Application of the Strain Index in multiple task Jobs. Applied Ergonomics, 40, 56-68.

Baybars, I. (1986). A survey of exact algorithms for the simple assembly line balancing problem. Management Science, 32, 909-932.

Battaïa, O., Dolgui, A. (2012). Reduction approaches for a generalized line balancing problem. Computers \& Operations Research, 39/10, 2337-2345.

Battaïa, O., Dolgui, A. (2013). A taxonomy of line balancing problems and their solutionapproaches. International Journal of Production Economics, 142/2, 259-277

Bautista, J., Pereira J. (2007). Ant algorithms for a time and space constrained assembly line balancing problem. European Journal of Operational Research, 177/3, 20162032.

Bautista, J., Cano, A., Alfaro, R. (2012). Models for MMSP-W considering workstation dependencies: A case study of Nissan's Barcelona plant. European Journal of Operational Research, 223/3, 669-679.

Becker, C., Scholl, A. (2006). A survey on problems and methods in generalized assembly line balancing. European Journal of Operational Research, 168, 694715.

Chica, M., Cordón, O., Damas, S., Bautista, J. (2010). Multiobjective constructive heuristics for the $1 / 3$ variant of the time and space assembly line balancing problem: ACO and random greedy search. Information Sciences, 180/18, 3465-3487.

Chica, M., Cordón, O., Damas, S., Bautista, J. (2011). Including different kinds of preferences in a multiobjective and algorithm for time and space assembly line balancing on different Nissan scenarios. Expert Systems with Applications, 38/1, 709-720.

Chica, M., Cordón, O., Damas, S., Bautista, J. (2012). Análisis del NSGA-II para el TSALBP-1/3 cuando existe variación de demanda en una producción mixta. VIIICongreso español sobre Metaheurísticas, Algoritmos evolutivos y bioinspirados. ISBN:978-84-615-6931-1.

McAtamney, L., Corlett, E. N. (1993). RULA: a survey method for the investigation of work-related upper limb disorders. Applied Ergonomics, 24/2, 91-99.

Otto, A., Scholl, A. (2011) Incorporating ergonomic risks into assembly line balancing European Journal of Operational Research, 212/2, 277-286.

Salveson, M.E. (1955). The assembly line balancing problem. Journal of Industrial Engineering, 6, 18-25.

Scholl, A., Fliedner, M., Boysen, N. (2010) Absalom: Balancing assembly lines with assignment restrictions. European Journal of Operational Research, 200, 688701.

Waters, T. R., Baron, S. L., Kemmlert, K. (1997). Accuracy of measurements for the revised NIOSH lifting equation. Applied Ergonomics , 29/6, 433-438. 